\title{
Welding sequence optimization of plasma arc for welded thin structures
}

\author{
M. B. Mohammed, W. Sun \& T. H. Hyde \\ Division of Materials, Mechanics and Structures, Faculty of Engineering, \\ University of Nottingham, UK
}

\begin{abstract}
Dividing the weld path into multiple seams that are welded sequentially in a specific order is one of the most important and cost effective distortion and residual stresses mitigation strategies. This is particularly important for welding fabrication of thin structures. The number of sequence options resulting from reordering seams increases explosively with the increase of the number of weld seams. Investigating all sequences using experimental, analytical or computational approaches requires substantial expenses in terms of time, resources and cost and in many situations is practically impossible. Therefore, welding industries tend to opt for small improvement and abandon the search for the absolute optimum welding sequence, e.g. the welding sequence that produces the absolute minimum welding induced distortion. This paper presents an optimization procedure to improving the effectiveness of the search for an optimized welding sequence. The optimization procedure is based on the principles of genetic algorithms (GA), in which the finite element (FE) analysis is used to produce the distortion information and direct the evolution of the GA within the optimization. The capability of the optimization procedure to identify an optimum welding sequence was demonstrated for the keyhole plasma arc welding (KPAW) of two Ti-6Al-4V thin structures, being a flat plate and a simplified replica of a portion of an aero-engine casing. The optimization procedure developed in this study was capable of minimizing the welding induced distortion by up to $55 \%$ and of improving the effectiveness of the search for an optimized welding sequence by up to $98 \%$.
\end{abstract}

Keywords: welding sequence, optimization, genetic algorithms, plasma arc welding, finite element modelling. 


\section{Introduction}

Welding generates distortion and residual stresses in structures as a result of the effects of shrinkage during cooling [1, 2]. These can lead to unacceptable inaccuracies and in-service defects, respectively [3]. A number of design-related and process-related mitigation strategies such as weld joint details, clamping, preheating and heat input are implemented to counteract this shrinkage effects [4].

Dividing the weld path into several seams that are welded sequentially in a specific order is one of the most important and cost effective distortion and residual stresses mitigation strategies [4]. The order (sequence) by which seams are welded alters the cooling patterns and as a result alters distortion and residual stresses evolution [3]. However, the number of sequences resulting from reordering weld seams increases explosively with increasing the number of weld seams which makes investigating all possible sequences using experimental, analytical or computational approaches substantially expensive [5]. Therefore, welding industries tend to opt for small improvement and abandon the search for the ultimate optimum welding sequence [4].

Previous research works have implemented various algorithms and methods in determining an optimum welding sequence. This includes the neural net alogrithm [5], the genetic and evolutionary alogrithm [1, 3, 6, 7] and the elstic net method [7]. These were all coded on the basis of the heuristic knowledge of welding experts in the robot welding industry while using FE modelling to calculate distortion. Distrotion was considered as the criterion determining the weld quality in these studies due to the fact that welding sequence has a minor effect on the resulting maximum residual stress values [1]. The heauristic knoweldge is however unique to the specific welding process of a specific structure which makes its emplementation across different welding processes and structures less resilient.

This paper presents the principles and application of the optimization procedure developed for improving the effectiveness of the search for an optimum welding sequence of thin structures. The optimization procedure is based on the principles of genetic algorithms (GA) and the finite element (FE) analysis software SYSWELD is used to produce the distortion information and direct the evolution of the GA in [8] within the optimization framework. The optimization procedure was demonstrated for the keyhole plasma arc welding (KPAW) of three thin Ti-6Al-4V structural components: a flat plate, a curved Tjoint and a simplified replica of a portion of an aero-engine casing.

\section{Principles of optimization using genetic algorithms}

Genetic algorithms searches for an optimized solution by the evolution of the existing solution using the mechanics of natural selection and natural genetics [9]. That is, if we think of each seam in the welding process as a gene and we think of any sets of seams as individuals composed of a string of genes, then GA applies genetic operations that results in the evolution of these individuals to 
produce fitter ones, i.e. optimized solutions. This is achieved by assigning fitness parameter to each solution and by selecting the existing optimum ones to go through the evolution process; also known as breeding. The three genetic operations associated with evolution are demonstrated below:

1. Crossover: Exchanging genes between the fittest breeding parents.

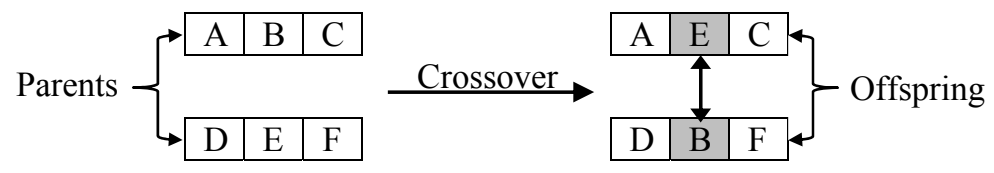

2. Mutation: Rearranging genes within each of the fittest breeding parents.

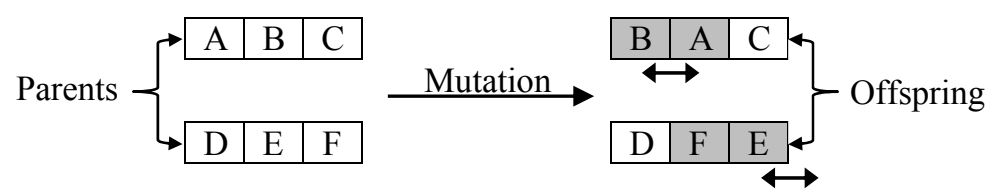

3. Reinsertion: This involves evolution by replacing parents with offspring.

GA is an effective tool to use in optimizing the distortion induced by seams welding sequence as the solution domain in GA is not limited by restrictive assumptions such as continuity and existence of derivatives.

\section{FE modelling of KPAW of thin structures}

The heat input required to establish the keyhole welding mode in plasma arc welding process is achieved by forcing plasma arc though a fine-bore nozzle at high velocities and high temperatures. KPAW process is superseded by laser and electron beam welding processes in terms of the power density these processes are capable of producing; KPAW process is however more cost effective and more tolerant of joint preparation [10].

The finite element models of the KPAW processes of two titanium alloy (Ti$6 \mathrm{Al}-4 \mathrm{~V}$ ) based thin structures were used to demonstrate the developed optimization procedure; these are a flat plate and a simplified replica of a portion of an aero-engine casing ().

The FE modelling of these thin structures was based on the work previously presented by Deshpande et al. [11] in which the KPAW processes modelling of bead on plate of the same material and thickness was developed and validated. The FE modelling was carried out using the commercially available welding specific software SYSWELD in which a 3D conical Gaussian heat source was used to model the heat input. The clamping conditions were defined by constraining the three velocity components $(U x, U y$ and $U z)$ of the nodes in the clamped areas on the thin structures. Heat transfer by convection and radiation were also accounted for. 
As illustrated in Figure 1(a), the flat plate has three seams while the aeroengine casing replica has four seams. Also, the FE modelling of the welding of the flat plate does not involve the joining of two separate plates; rather, the heat source representing the welding torch was applied along the mid-section of a single plate. Hence, distortions in the flat plate are purely generated due to the contribution of heating and cooling cycle in addition to clamping conditions. In the case of the simplified replica of a portion of an aero-engine casing the FE modelling of the welding involves the joining of the outer ring with the inner structure represented respectively by the solid and dotted lines as shown in Figure 1(b). This was incorporated in the FE model by adding a very thin layer $(0.25 \mathrm{~mm})$ of "chewing gum elements" at the interface between the two

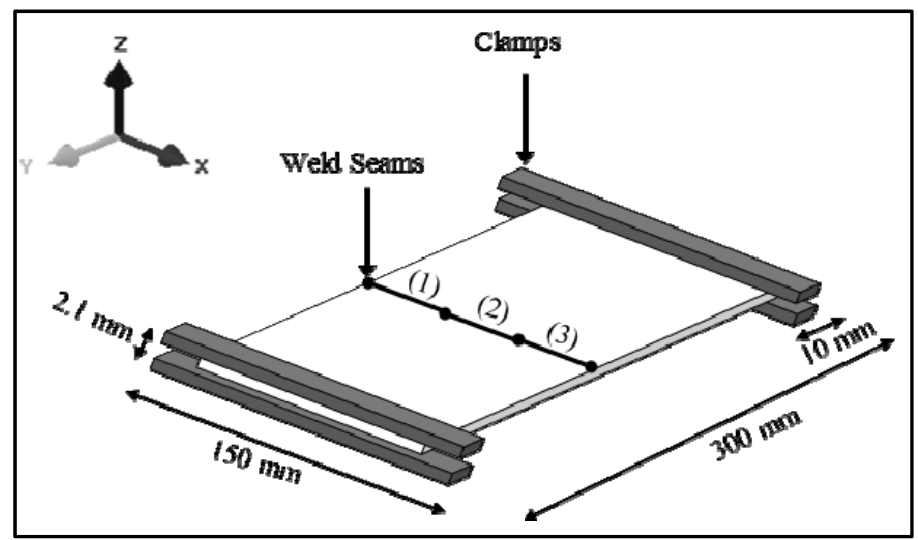

(a)

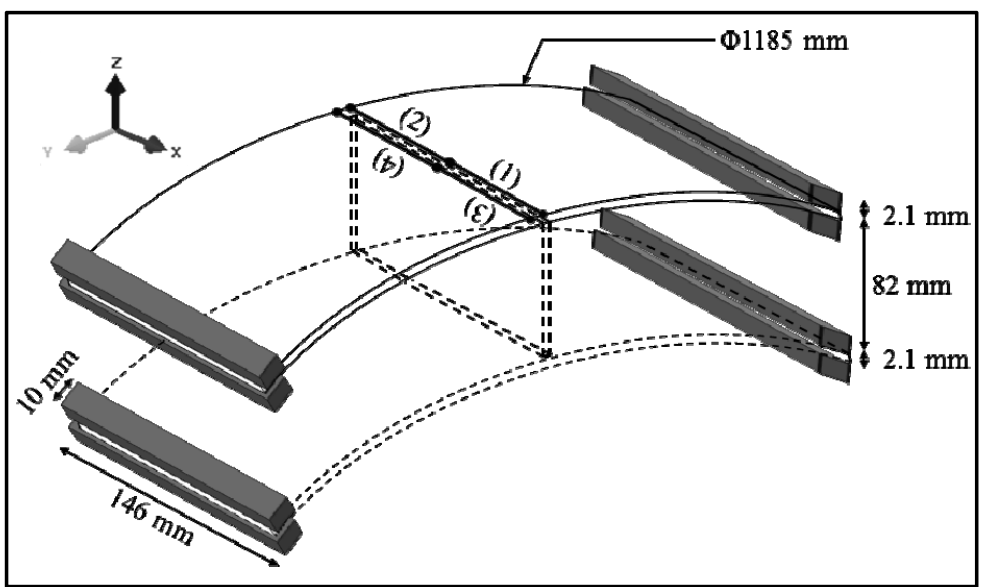

(b)

Figure 1: $\quad$ FE modelling set-up for the KPAW of Ti-6Al-4V (a) Flat plate; (b) Simplified replica of a portion of an aero-engine casing. 
structures (Figure 2). These chewing gum elements have no stiffness, hence the name, and it undergoes transition from the no stiffness state to the state of taking on the mechanical behaviour of the Ti-6Al-4V once their temperature exceeds the melting point. This allows for the representation of the local mechanical interlocking between the welded structures as a result of the occurrence of fusion.

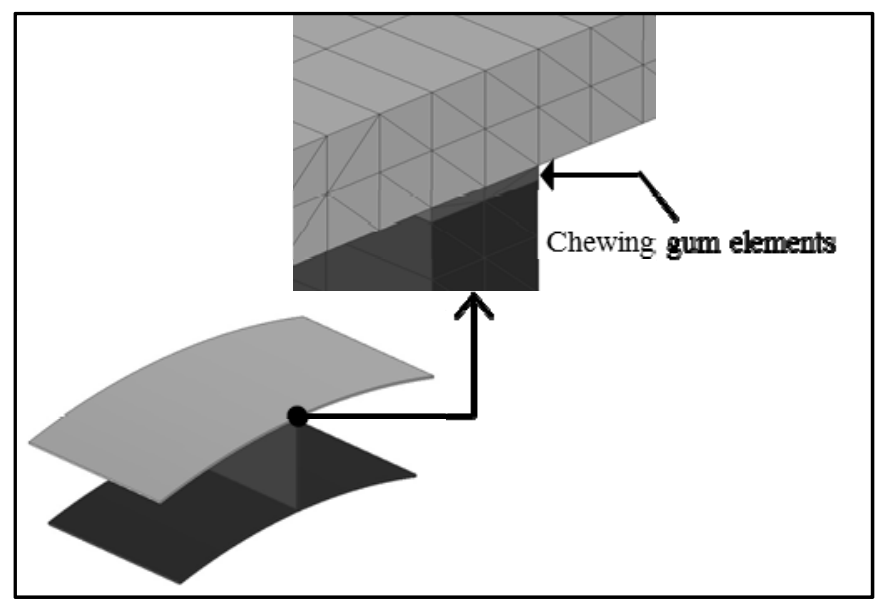

Figure 2: Interfacing welded structure using chewing gum elements to represent fusion.

\section{Definition of welding sequence problem}

The seams in the FE modelling of the flat and the simplified replica of a portion of an aero-engine casing are of equal length in both cases. While the direction of welding each seam can vary, each seam can be welded only once. Therefore, each welding sequence consists of a string containing all seams. The total number of possible welding sequences is the number of combinations that can be made by rearranging the orders and directions of seams. This is demonstrated in (Table 1) for the flat plate. As can be seen in Table 1, the order of welding a seam is represented by its position in the sequence; being $1^{\text {st }}, 2^{\text {nd }}, 3^{\text {rd }}$, etc. and the direction of welding a seam is represented by the $(+)$ and $(-)$ signs.

The total number of all possible welding sequences can be obtained using Equation (1) introduced in $[1,12]$ in which $d$ is the number of directions for each seam ( 2 directions) and $s$ is the total number of seams (3 and 4). As a result, there is a total of 48 and 384 possible welding sequences for the flat plate and the simplified replica of a portion of an aero-engine casing.

$$
d^{s} \times s !
$$


Table 1: Example of welding sequences resulting from rearranging the orders and directions of seams in the flat plate.

\begin{tabular}{|c|c|c|c|c|c|c|}
\hline \multirow{2}{*}{$\begin{array}{c}\text { Sequence } \\
\text { No. }\end{array}$} & \multicolumn{3}{|c|}{ Seams Welding Orders and Directions } & \multirow{2}{*}{\multicolumn{3}{|c|}{ Schematic }} \\
\hline & $1 \mathrm{st}$ & $2^{\text {nd }}$ & $3 \mathrm{rd}$ & & & \\
\hline 1 & +1 & +2 & +3 & 1 st & $2^{\text {nd }}$ & $3^{\text {rd }}$ \\
\hline 2 & -2 & +1 & +3 & $2^{\text {nd }}$ & $1^{\text {st }}$ & $3^{\text {rd }}$ \\
\hline 3 & -3 & -2 & -1 & $3^{\text {rd }}$ & $2^{\text {nd }}$ & 1 st \\
\hline
\end{tabular}

Identifying the absolute optimum welding sequence, i.e. the welding sequence that produces the absolute minimum distortion, can only be achieved by modelling all possible sequences. This is expensive in terms of computational efforts, time and the costs associated with it. Hence, there is a need for an optimization procedure to be employed in order to improve the effectiveness of identifying a welding sequence that can produce a minimum distortion.

\section{Structure of welding sequence optimization procedure}

The structure of the optimization procedure is composed of two interacting elements; genetic algorithms to optimize the search of an optimum welding sequence and FE modelling to assess and direct the search for an optimum welding sequence. The interaction between GA and FE modelling is developed on a MATLAB platform using the structure shown in Figure 3. As Figure 3 shows, GA is initially coded to create an initial population (parent sequences)

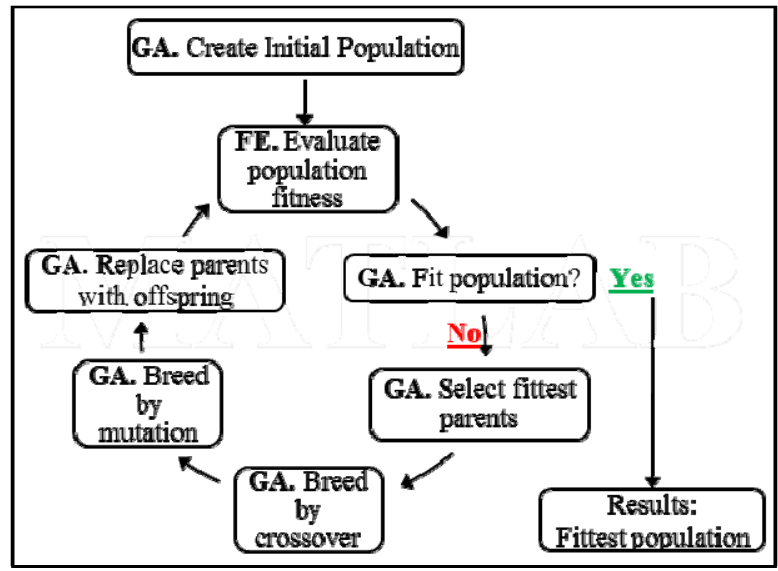

Figure 3: Structure of optimization procedure developed on MATLAB platform. 
which will go through the process of genetic (seam) evolution. These initial parent sequences were coded to carry all genes in order to give each gene equal opportunity to survive and make it to the next generation. The parent sequences coded for the three seams in the flat plate and for the four seams in the simplified replica of a portion of an aero-engine casing are shown in Table 2.

Table 2: $\quad$ Parent sequences coded to bare all seams.

\begin{tabular}{l|c|c|c|c|c|c|c|c|c|}
\hline & \multicolumn{4}{c}{ Parent (1) } & \multicolumn{4}{c}{ Parent (2) } \\
\hline Three Seams & +4 & +2 & +3 & & -1 & -2 & -3 \\
\hline Four Seams & +1 & +2 & +3 & +4 & -1 & -2 & -3 & -4 \\
\hline
\end{tabular}

The fitness of each of the parent sequences is then evaluated by calculating the distortion introduced by each of the parent sequences to the welded structure using FE modelling. This is achieved by the MATLAB code developed to modify the FE modelling input files to reflect the welding sequence created by GA and to feed the distortion results back to it. Because the distortion in the zdirection is dominant as shown in Figure 4 for the flat plate, the value of the objective function to be minimized in this optimization procedure was defined as the maximum displacement in the z-direction $(U z)$ as shown in Equation (2) where $i$ is the $i^{\text {th }}$ welding sequence.

$$
\begin{gathered}
\operatorname{Min} \rightarrow \operatorname{Obj} \cdot \operatorname{Fun}(1)=\max |U z| \\
\vdots \\
\operatorname{Min} \rightarrow \operatorname{Obj} \cdot \operatorname{Fun}(i)=\max |U z|
\end{gathered}
$$

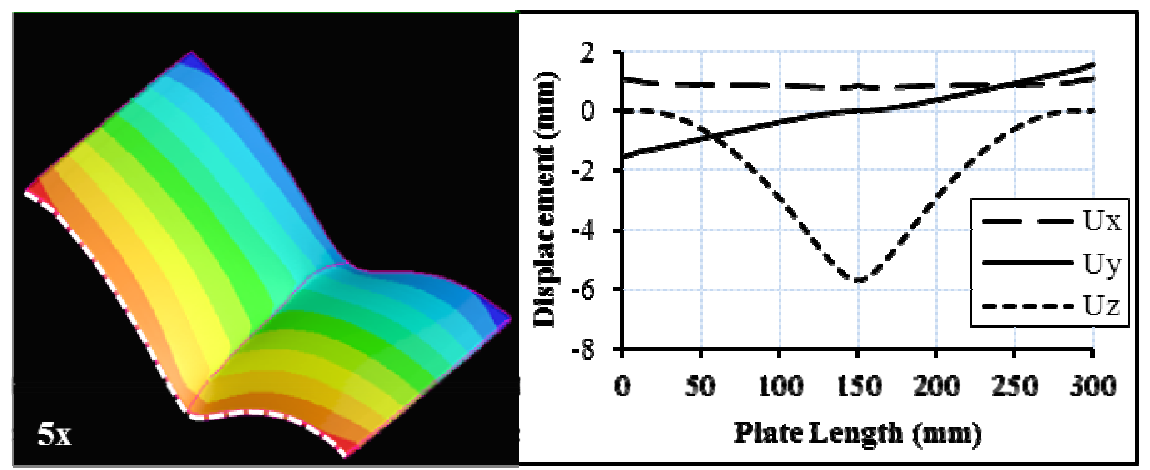

Figure 4: Displacements profiles along the white dotted line in the $\mathrm{x}, \mathrm{y}$ and $\mathrm{z}$ directions in the flat plate.

The parent sequences are then assigned a rank on the basis of the distortion they induce; that is higher rank for minimum distortion. A new welding sequence (offspring welding sequence) is then generated as a result of the breeding of the 
parent sequences by single gene crossover and single gene mutation. The fitness of the new offspring sequence is then assessed and a rank is assigned to it. The new offspring welding sequences replaces the parent sequence that has lower rank in order to ensure that only fitter parents are contributing to the evolution of the new generations, i.e. new optimized sequences.

The optimization procedure described above continues until the optimization criterion described in Equation (3) is met, where $\beta=0.4$; this minimizes the distortion by $40 \%$ relative to the maximum distortion identified during the optimization procedure. The value of $\beta=0.4$ was found to be effective in identifying an optimum welding sequence for the welds presented in this paper.

$$
\frac{\min (\operatorname{Obj} \cdot \operatorname{Fun}(1 \ldots i))}{\max (\operatorname{Obj} \cdot \operatorname{Fun}(1 \ldots i))} \leq \beta
$$

\section{Results and discussions}

Table 3 and Figure 5 show the results of the optimization procedure for the flat plate. As can be seen, an optimized welding sequence $(-1-3+2)$ was identified in accordance with the optimization criterion described in Equation (3) in the fifth iteration. The optimized welding sequence produced a maximum absolute

Table 3: Evolution of the 3 seams welding sequence of flat plate in optimisation.

\begin{tabular}{|c|c|c|c|c|}
\hline Iteration & \multicolumn{3}{|c|}{ Weld Sequence } & $|\mathrm{Uz}|(\mathrm{mm})$ \\
\hline 1 & -1 & -2 & -3 & 8.34 \\
2 & +2 & +1 & -3 & 6.98 \\
3 & +2 & -1 & -3 & 7.65 \\
4 & +2 & -3 & -1 & 8.01 \\
5 & -1 & -3 & +2 & 3.76 \\
\hline
\end{tabular}

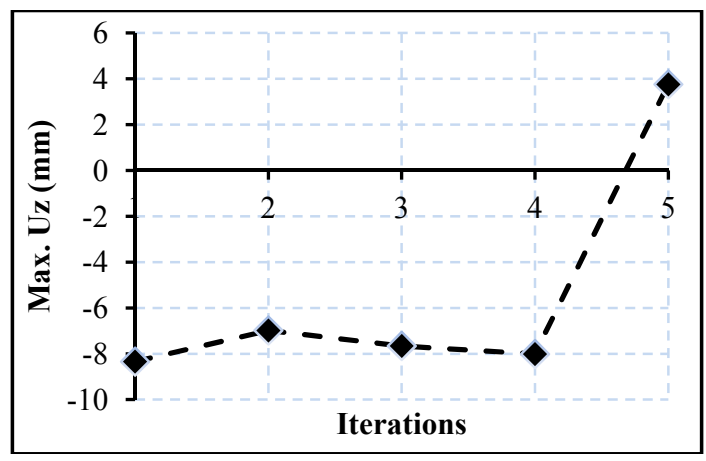

Figure 5: Maximum distortions in z-direction for the 3 seams welding sequence optimization of flat plate. 
distortion of $3.76 \mathrm{~mm}$ which is less by about $55 \%$ of the maximum distortion that was produced by sequence $(-1,-2,-3)$ in the first iteration. In this case, the optimization procedure was able to minimize distortion by about $55 \%$ by the FE modelling of 5 sequences out of a total of 48 possible sequences which saves about $90 \%$ of the time required for the FE modelling of all sequences.

The results of the optimization procedure for the simplified replica of a portion of an aero-engine casing are shown in Table 4 and Figure 6 . The optimum welding sequence $(+1-4+2-3)$ was identified in the ninth iteration and produced a maximum distortion of $1.27 \mathrm{~mm}$ which is about $40 \%$ less than the distortion produced in the eighth iteration. Identifying an optimized welding sequence in the ninth iteration implies that about $98 \%$ of time required for the FE modelling of all possible welding sequences (384 sequences) was saved while achieving $40 \%$ reduction in welding induced distortion.

Table 4: $\quad$ Evolution of the 4 seams welding sequence of the simplified replica of a portion of an aero-engine casing.

\begin{tabular}{|c|c|c|c|c|c|}
\hline Iteration & \multicolumn{4}{|c|}{ Weld Sequence } & $|\mathrm{Uz}|(\mathrm{mm})$ \\
\hline 1 & +2 & -4 & -3 & +1 & 1.96 \\
2 & +1 & -4 & -3 & -2 & 1.97 \\
3 & +1 & -4 & -2 & -3 & 1.88 \\
4 & +1 & -4 & -2 & +3 & 2.05 \\
5 & +2 & -3 & -1 & +4 & 1.99 \\
6 & +1 & -2 & +4 & -3 & 1.61 \\
7 & +1 & +4 & +2 & +3 & 2.00 \\
8 & +2 & -4 & -1 & -3 & 2.11 \\
9 & +1 & -4 & +2 & -3 & 1.27 \\
\hline
\end{tabular}

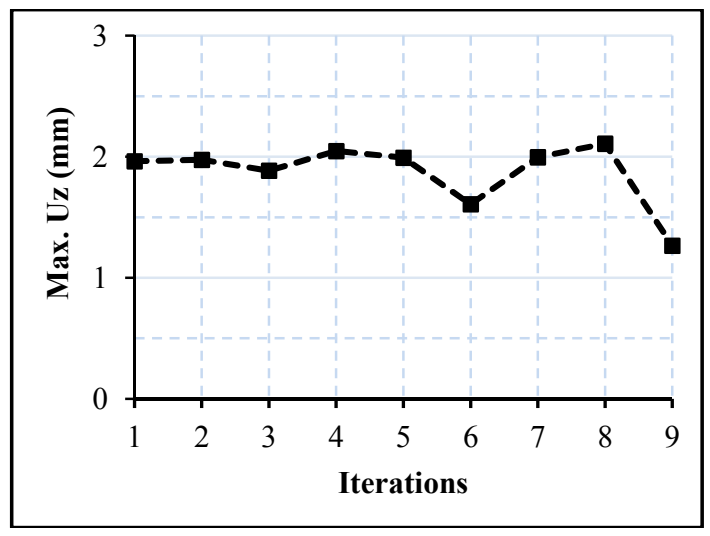

Figure 6: Maximum distortions in z-direction for the 4 seams welding sequence optimization of simplified replica of a portion of aeroengine casing. 
Figure 7 shows a comparison between the distortions resulting from using continuous welding (no seams) and using optimized welding sequence for both of the flat plate and the simplified replica of a portion of an aero-engine casing. The comparison clearly shows that continuous welding did not result in reduced distortion hence validating the concept of dividing weld bead into several seams to minimize distortion.

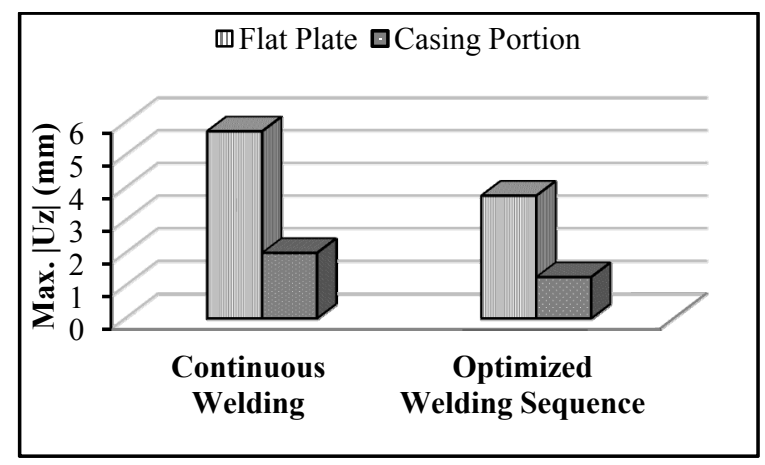

Figure 7: Comparison between distortions induced by continuous welding and the optimized welding sequences.

\section{Conclusions}

Welding induced distortion can be minimized by dividing the weld bead into small seams that are welded in a specific order. Identifying the absolute optimum welding sequence requires investigating all possible welding sequences which is expensive in terms of computational efforts, time and cost. An optimization procedure that was developed on the basis of the integration of GA and FE modelling in a MATLAB platform is proposed in this paper to improve the effectiveness of the search for an optimized welding sequence, i.e. the welding sequence that minimizes welding induced distortion. The optimization procedure was applied in the FE modelling of a KPAW of Ti-6Al-4V flat plate that has three seams and a simplified replica of a portion of an aero-engine casing that has 4 seams. The optimization procedure identified an optimum welding sequence that minimized distortion by about $55 \%$ in the fifth iteration for the former and about $40 \%$ in the ninth iteration for the latter. As a result a saving of up to $90 \%$ and $98 \%$ of FE modelling time has been achieved for both welding models, respectively. It was demonstrated that the optimized welding sequences minimized distortion in comparison to continuous welding.

The optimization procedure presented in this paper can be conveniently applied to other similar welding processes, such as laser and electron beam welding, and can also be coded to minimize other structural parameters such as residual stresses. 


\section{Future work}

Figure 8 shows the full-size simplified replica of the aero-engine casing from which the portion presented in this paper was extracted. As can be seen in the figure, the full-size replica is composed of 12 portions. The distortion optimization of one portion presented in this paper is considered as a local optimization. Future work will focus on developing global optimization of welding induced distortion. That is, optimizing the order by which the 12 portions are welded to minimize the global distortion of the full-size structure. This will improve the robustness of the optimization procedure in solving practical welding induced distortion problems required in welding industries.

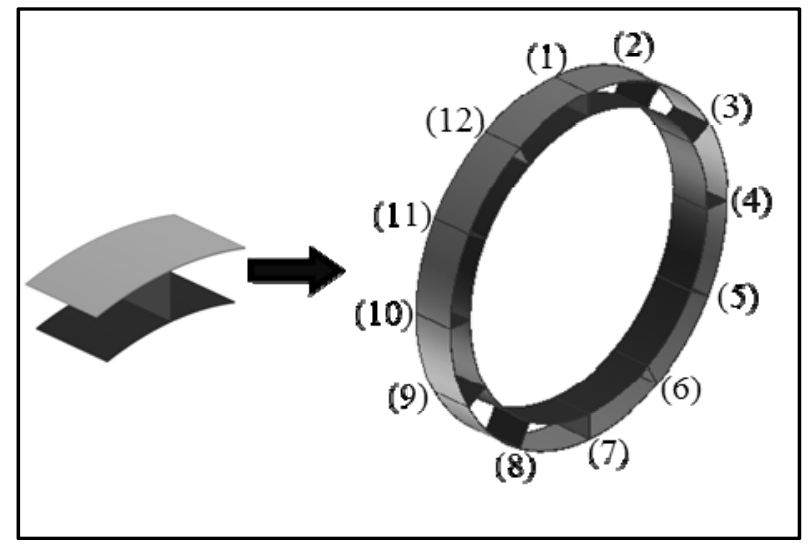

Figure 8: Full-size simplified replica of an aero-engine casing.

\section{Acknowledgements}

The authors of this paper would like to acknowledge the EPSRC for their financial support of the research work through the Nottingham Innovative Manufacturing Research Centre (NIMRC) at the University of Nottingham.

\section{References}

[1] Kadivar, M.H., K. Jafarpur, and G.H. Baradarn, Optimizing welding sequence with genetic algorithm. Journal of Computational Mechanics, 2000. 26: p.514-519.

[2] Tsai, C.L., S.C. Park, and W.T. Cheng, Welding distortion of a thin-plate panel structure. Welding Research Supplement, 1999: p.156-165.

[3] Damsbo, M. and P.T. Ruhoff, An evolutionary algorithm for welding tasl sequence ordering. AISC'98, LNAI, 1998. 1476: p.120-131. 
[4] Nnaji, B.O., D. Gupta, and K.Y. Kim, Welding distortion minimization for an aluminum alloy extruded beam structure using a 2D model. Transactions of the ASME, 2004. 126: p.52-63.

[5] Fukuda, S. and K. Yoshikawa, Determination of welding sequence: a neural net approach. Journal of Engineering Analysis with Boundary Elements, 1991. 7(2): p.78-82.

[6] Kim, K.Y., D.W. Kim, and B.O. Nnaji, Robot arc welding task sequencing using genetic algorithms. IIE Transactions, 2002. 34: p.865-880.

[7] Yang, H. and H. Shao, Distortion-oriented welding path optimization based on elastic net method and genetic algorithm. Journal of Materials Processing Technology, 2009. 209: p.4407-4412.

[8] Hartmut, P., GEATbx - Genetic and Evolutionary Algorithm Toolbox for use with Matlab, 1994-2006.

[9] Goldberg, D.E., Genetic Algorithms in Search, Optimization, and Machine Learning1989: Addison-Wesley Publishing Company, Inc. 412.

[10] Wu, C.S., Q.X. Hu, and J.Q. Gao, An adaptive heat source model for finiteelement analysis of keyhole plasma arc welding. Computational Materials Science, 2009. 46: p.167-172.

[11] Deshpande, A.A., A.J. Short, W. Sun, D.G. McCartney, L. Xu, and T.H. Hyde. Parameter idnetification for stable keyhole plasma arc welding of a Ti-6Al-4V sheet. in AeroMat 2010. Bellevue, WA, USA.

[12] Voutchkov, I., A.J. Keane, A. Bhaskar, and T.M. Olsen, Weld sequence optimization: The use of surrogate models for solving sequential combinatorial problems. Journal of Computer Methods in Applied Mechanics and Engineering, 2005. 194: p.3535-3551. 